\title{
Research and application on innovative technologies for digital construction of network floor
}

\author{
Shao Junyi ${ }^{1, a}$, Song Yanlei ${ }^{1, b}$, Cao Xuemei ${ }^{1}$ and $\mathrm{Wu} \mathrm{Di}^{1}$ \\ ${ }^{1}$ Qingdao Technological University, 266520 Qingdao, Shandong, China
}

Keywords: network floor, CAD secondary typesetting, laser level levelling, shock pads fine tuning, computer code nesting.

\begin{abstract}
With the rapid development of new technologies and new building materials, new network floor as a type of new building materials has been widely used in ground projects, owing to its wiring flexible, simple and practical, easy maintenance, and environmental friendly features. However, there are problems in the traditional construction process of network floor, like unreasonable arrangement, poor flatness and the waste of side plate cutting. To address these issues, this paper firstly proposes three innovative technologies for the comprehensive control of the implementation of network floor project, including CAD secondary typesetting and layout technology, precision laser level levelling + shock pads fine tuning technology, and computer code nesting of side plate technology. Furthermore, a complete set of construction technology processes has been presented. Finally, the economic and social benefits of these innovative technologies have been proved by the engineering project.
\end{abstract}

\section{Introduction}

Ground engineering is an important part of interior decoration project. With the rapid development of economy and technologies, the requirements on office environment are increasingly high. The application of various intelligent systems and terminal equipment increase the difficulty of interior surface wiring project greatly, which affect the clean and beautiful of office environment. However, the appearance of network floor is a good solution to this problem. Network floor is a type of new floor that specific application in the modern office space. Compared to the traditional ground project, the network floor has a series of advantages, such as wiring flexible, simple, practical, convenient maintenance, green environmental protection and so on. Network floor is replacing the traditional brick and stone floor gradually, which is widely used in office buildings, office buildings, intelligent buildings and other places with high standard on communication system ${ }^{[1]}$.

However, due to the lack of research on construction technology of network floor, the traditional construction methods mainly exists unreasonable arrangement, poor flatness, the waste of side plate cutting and other quality problems ${ }^{[2]}$, which affected the use of network floor greatly. Therefore, a comprehensive analysis of co-ordination arrangements and technological innovation should be taken into account, which will greatly accelerate the further use and development of network floor.

aShao Junyi :qdlgdx@126.com b Song Yanlei :414809456@qq.com 


\section{Construction Technological Processes Of Innovative Technologies}

To achieve the high-quality goal of network floor, this paper proposes three innovative technologies for the comprehensive control of the implementation of network floor project, including CAD secondary typesetting and layout technology, precision laser level levelling + shock pads fine tuning technology and computer code nesting of side plate technology, which will solve the above problems effectively.

In order to ensure the smooth implementation of the innovative technologies, this paper presents a complete set of construction technology processes. The main construction process including CAD secondary typesetting, surface cleaning up, dividing line, laser level fixed floor bracket, network floor installation, cushion fine-tuning, computer code ${ }^{[3]}$. Detail in Figure1.

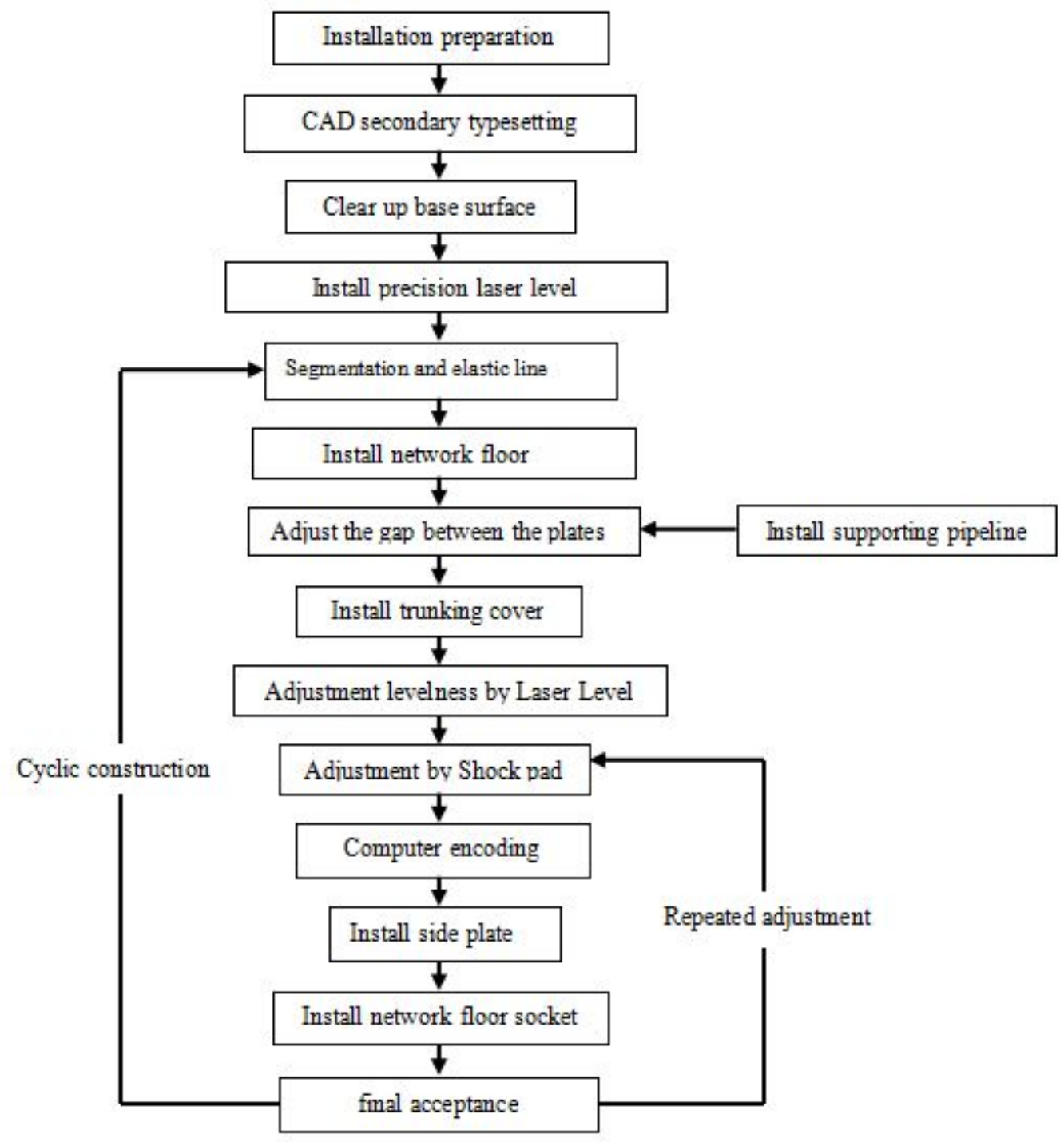

Figure 1. Construction technological processes of innovative technologies 


\section{The main technical operation points of innovative technologies}

\subsection{Installation preparation}

\subsubsection{Technical preparation}

(1) Each layer of the ground shall have been accepted according to the design requirements.

(2) The drawings required for the construction of the ground shall be properly prepared.

(3) DSJ3 precision laser level, DT-2A laser theodolite have been tested and corrected, the accuracy can reach the construction requirements.

\subsubsection{Material requirement}

( 1 ) Network floor: The surface layer of material must meet the design requirements. The board requires no damage and small chromatic aberration.

(2) Accessory bracket: Anchor bolts, socket plate, cushion and other accessories shall comply with the design requirements, ensuring the size is accurate. The installation schematic diagram of network floor is shown in figure 2 .

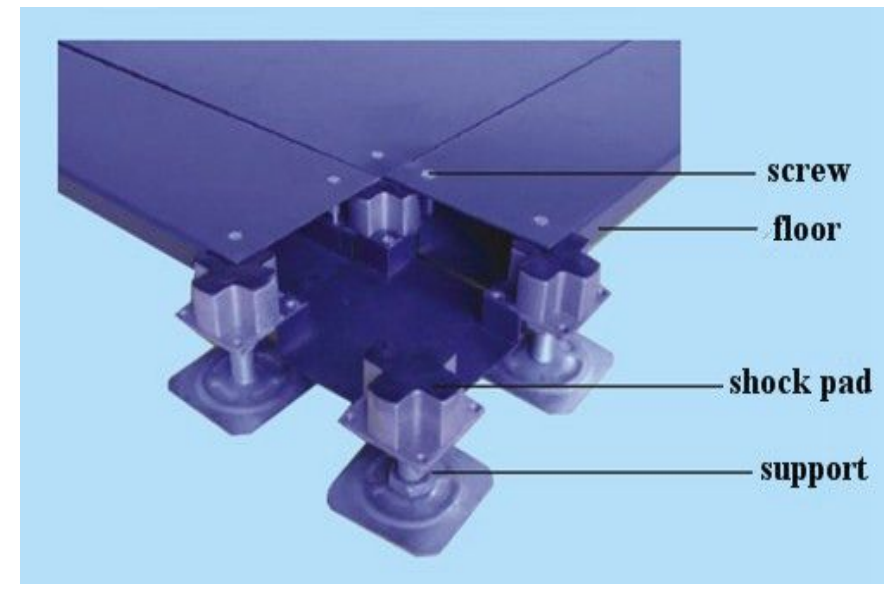

Figure 2. The installation schematic diagram of network floor

\subsection{CAD secondary typesetting}

(1) Basic principles: The cutting quantity of network floor plate and trunking is least, the interface between the plate and the plate is tight.

(2) By measuring the actual size of all the different room, according to the network floor plate and cover dimensions, taking into account the impact of the edge of the wall and protruding columns, typesetting all the plates of networks floor by using CAD and coding all the plates to achieve efficient splice.

(3) All standard plates and profiled plates are numbered, positioning and installation in accordance with the number strictly. Taking one of the standard rooms as an example, the detail of CAD secondary typesetting is shown in Figure 3. 


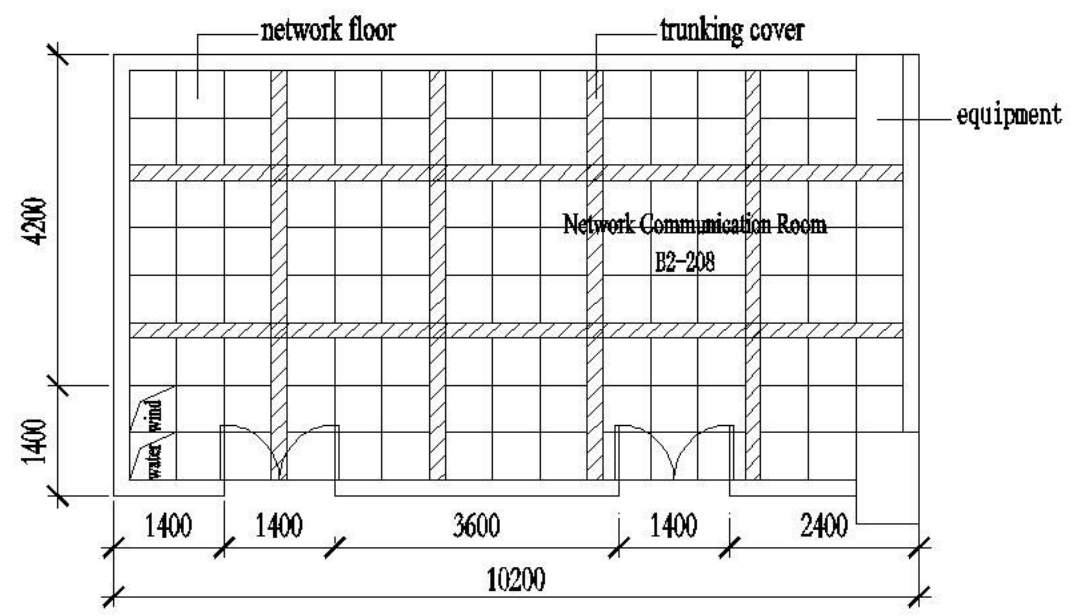

Figure3. The detail of CAD secondary typesetting

\subsection{Clear up base surface}

Network floor's base surface should meet the following requirements: base surface is concrete floor, which have been pressed and smooth, grass-roots should be fully dry, moisture content should not be greater than $5 \%$, the base surface should be clean, no cracks, no peeling, no pockmarked face and other phenomena ${ }^{[4]}$. Check flatness should meet the following requirements: the error does not exceed $\pm 3 \mathrm{~mm}$ within 3 meters.

\subsection{Segmentation and elastic line}

(1) Basic principles: According to the CAD secondary layout diagram, room size, equipment layout and the module of network floor, determine the laying direction and the starting point.

( 2 ) Selecting a wall as the reference surface, using the DT-2A laser theodolite to determine a baseline, which is $595 \mathrm{~mm}$ away from the reference surface. The baseline is treated as the transverse control line of mounting direction.

(3) Based on the position of the transverse baseline, determine a vertical line, which is also $595 \mathrm{~mm}$ away from the adjacent wall, the baseline is treated as the vertical control line of mounting direction. The transverse control line and vertical control line are shown in Figure 4.

( 4 ) In accordance with the above-identified transverse control line and vertical control line, snapping mesh division on the basal surface, finally formation square grid with correct size.

\subsection{Determine the installation height of network floor}

(1) According to the overall layout of the room and CAD secondary layout diagram, setting DSJ3 precision laser level at the intersection of reference line and vertical line, which mainly used to control the flatness of plate and trunking cover.

( 2 ) Operating DSJ3 precision laser level to project the elevation control line at the four wall of interior room, which is help to control the subsequent construction.

( 3 ) Using laser level to find the highest and lowest points of whole room, calculating the average between ground and network floor, this figure is treated as the installation height of network floor ${ }^{[5]}$. 


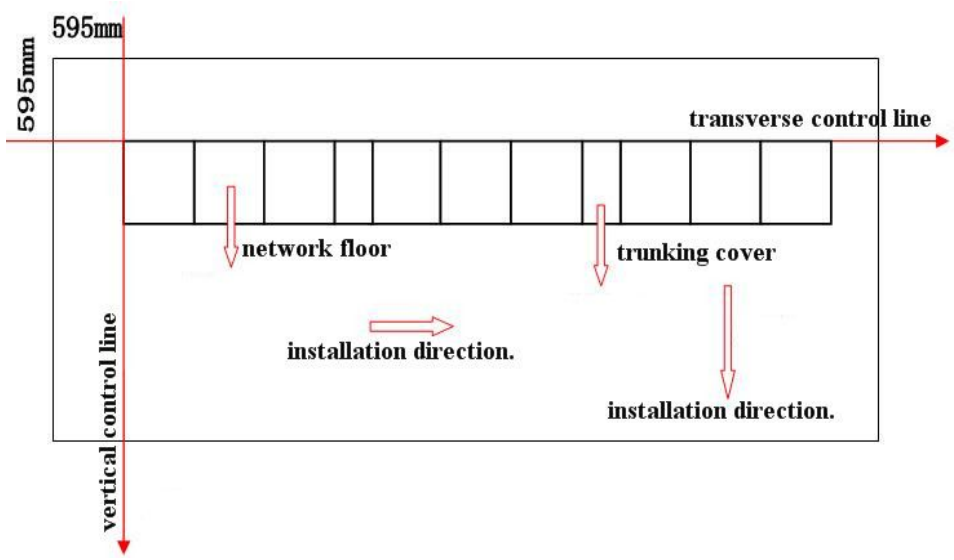

Figure4. The schematic diagram of transverse control line and vertical control line

\subsection{Installation adjustable bracket by using laser theodolite}

(1) According to the square grid that has been completed, determine the construction reference point.

(2) Starting from the construction reference point, installation adjustable bracket in turns at the intersection of the cross square grid, the bracket including its supporting bearing, shock pad, bolts and anchor bolts, using anchor bolt to fix the bottom of bracket.

( 3 ) Based on the infrared ray that projected by DSJ3, overall control of the installation level of bracket, installation level should meet the following requirements ${ }^{[6]}$ : The error should not exceed \pm $1 \mathrm{~mm}$ in the $3 \mathrm{~m}$ range, and the error of entire room should not exceed $\pm 2 \mathrm{~mm}$.

\subsection{Installation plate of network floor}

(1) Installation plate of network floor from the construction reference point, the installation height of plate should be consistent with the elevation control line, using DSJ3 laser level to check the flatness of plate.

( 2 ) When the first piece of the plate fixed in place, installation plate of network floor in the direction of transverse control line and vertical control line.

( 3 ) In the installation process of network floor, making full use of the DSJ3 laser level to implement global and repeated testing, comprehensive control the installation flatness. At the same time, use the pad to adjust flatness of network floor slightly.

\subsection{The side plate encoding and installation}

Due to the limitation of network floor processing module, at some special parts of room, such as the edge of the wall and projecting column, the size of reserved is less than a standard network floor plate. Therefore, it needs to apply the side plate implement installation. The construction technology of the side plate is as follows:

Based on the size of the reserved, coding into the computer unified, shaping and cutting on site, ensuring the overall cutting optimization. At the same time, it needs to number the side plate one by one, and all of the cutting edges should implement anti-rust treatment by using epoxy paint, and to ensure that the side plates have a better perception. There are two main treatment options of side plate $^{[7]}$, specifically shown in Figure5. 

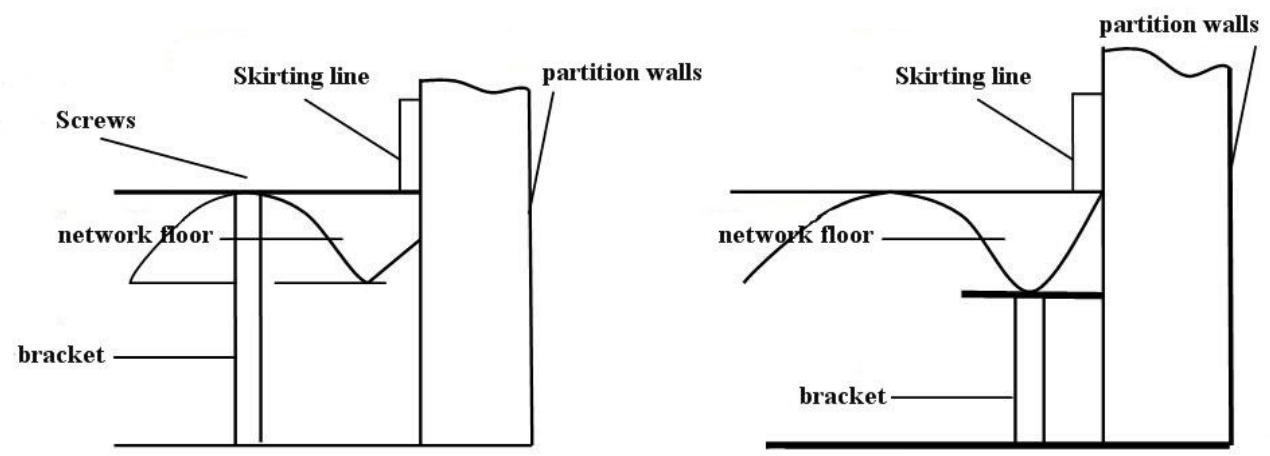

Figure5. The main treatment options of side plate

\section{Application examples}

The integrated band innovative construction technology has been applied to a project of Qingdao Development Zone. The project is an office building with refined decoration and total construction area of the office building is 34832.32 square meters, the ceiling project adopt the integrated band.

It has been proven through practice that the proposed integrated band digital construction technologies can achieve twin objectives of saving and aesthetics. The technologies can ensure the rationality of the layout of the plate, save the amount of plate and improve installation efficiency of network floor. Not only can these technologies reduce the cost of quality, but also they can improve the social benefits.

\section{Conclusions}

Network floor as a type of new building materials has been widely used in ground projects, this paper proposes three innovative technologies for the comprehensive control of the implementation of network floor project, which solve the problems arise in the construction process effectively, such as unreasonable arrangement, poor flatness and the waste of side plate cutting. At the same time, these innovative technologies accelerate the construction progress, improve installation quality and also extend the service life of the floor, which have notable economic benefits and social benefits.

The key technologies of this paper proposed are reliable. The process of construction is environmental friendly and energy-saving, which winning the high user satisfaction. These innovative technologies will help to further promote the popularization and application of network floor.

\section{References}

1. Wen-liang Lv. Applications technology of steel and space network floor [J]. Construction Technology,2008,37(2):99-102.

2. Hai-fengYang. The application of OA network floor in a certain project [J]. Shanxi Architecture,2015,41(18):95-96.

3. Jin-song Shan, Liang Huang, Chang-ping Liu, etc. Installation and application of modular net floor with trunkings [J]. Building Technique Development,2013,40(7):60-63.

4. Bao-lin Wang. Construction of anti-static floor [J]. Shanxi Architecture,2013,39(24):118-119.

5. Han-chen Liu, Wen-qing Xiao. Study on construction technology and quality control of large area full steel OA network floor [J]. Guangdong Building Materials,2011,(7):14.

6. Wei Liu. Discussion on the application of steel OA anti-static network floor in building engineering [J]. Construction Technique,2014,10:39-40.

7. Rui-nian Wang, Da-zhi Yuan,Yue Deng. Construction technology of all steel anti static network floor [J]. Tianjin Construction Science and Technology,2014,24(1):12-13. 